\title{
Atmospheric Science Measurements by the EOS Geoscience Laser Altimeter System
}

\author{
James Spinhime \\ NASA Goddard Space Flight Center \\ Laboratory for Atmospheres/912 \\ Greenbelt, MD 20771 \\ 301-614-6274 \\ spinhimeiogsfo.nasa.gov
}

Scheduled for Launch in July 2001, the Geoscience Laser Altimeter System (GLAS) is to be the first satellite instrument to provide full global lidar profiling of clouds and aerosol in the earth's atmosphere. GLAS is an EOS program instrument that is on its own satellite, now called the Ice, Cloud and land Elevation Satellite. The instrument is both a surface laser ranging system and an atmospheric profiling lidar. A most important surface measurement for the instrument is to study the change in the mass balance of the polar ice sheets by measuring the change in regional altitudes to an accuracy of $1.5 \mathrm{~cm}$ per year. The strategy to combine the surface measurement with a cloud and aerosol lidar profiling mission is based on the compatibility of the altimetry instrument requirements with those for the required lidar measurements.

The primary atmospheric science goal of the GLAS cloud and aerosol measurement is to determine the radiative forcing and vertically resolved atmospheric heating rate due to cloud and aerosol by directly observing the vertical structure and magnitude of cloud and aerosol parameters that are important for the radiative balance of the earth-atmosphere system, but which are ambiguous or impossible to obtain from existing or planned passive remote sensors. A further goal is to directly measure the height of atmospheric transition layers (inversions) which are important for dynamics and mixing, the planetary boundary layer and lifting condensation level. The GLAS atmospheric measurements have the requirement to profile all radiatively significant cloud and aerosol up to the limit of signal attenuation. The definition of the atmospheric measurement requirements and performance for GLAS are the result of extensive experience and analysis with existing airborne experimental data since 1980 (Spinhirne et al., 1982 and following). The basic parameterization of the measurement requirement is in terms of the required measurement accuracy and spatial resolution for the given range of observed backscatter cross section. The stated requirements are listed in table 1

Table 1. GLAS Requirement for Cloud and Aerosol Profiling

\begin{tabular}{|c|c|c|c|c|c|}
\hline Measurement & $\begin{array}{r}\text { Spatial } \\
\text { Horizontal }\end{array}$ & $\begin{array}{c}\text { Requirement } \\
\text { Vertical }\end{array}$ & $\begin{array}{c}\text { Cross Section } \\
\text { Range (m-sr) }\end{array}$ & $\begin{array}{c}\text { Opt. Thick. } \\
\text { per km (aprx). }\end{array}$ & $\begin{array}{c}\text { Accuracy } \\
\text { Requirement }\end{array}$ \\
\hline Dense Clouds & $0.2 \mathrm{~km}$ & $100 \mathrm{~m}$ & $10^{-4}-10^{-2}$ & $2-200$ & $10 \%$ \\
\hline Cirrus & $1-20 \mathrm{~km}$ & $300 \mathrm{~m}$ & $10^{-6}-10^{-4}$ & $.02-2$ & $10 \%$ \\
\hline Thin Cirrus & $20-50 \mathrm{~km}$ & $300 \mathrm{~m}$ & $10^{-7}-10^{-5}$ & $.002-.2$ & $10 \%$ \\
\hline PBL Aerosol & $1-150 \mathrm{~km}$ & $150 \mathrm{~m}$ & $10^{-7}-10^{-4}$ & $.005-3$ & $10 \%$ \\
\hline Upper Trop. Aerosol & $100-1000 \mathrm{~km}$ & $500 \mathrm{~m}$ & $10^{-7}-10^{-6}$ & $.005-.03$ & $10 \%$ \\
\hline Strat Aerosol & $1000 \mathrm{~km}$ & $1000 \mathrm{~m}$ & $10^{-7}-10^{-6}$ & $.005-.05$ & $2 \%$ \\
\hline Molecular Return & $5000 \mathrm{~km}$ & $2000 \mathrm{~m}$ & $10^{-8}-10^{-7}$ & & $2 \%$ \\
\hline Solar Background & $1 \mathrm{~km}$ & & & & $1 \%$ \\
\hline
\end{tabular}

In the table the range of optical thickness is included to illustrate the sensitivity of the backscatter lidar for thin cloud and aerosol. The measurement approach relies on along 
track averaging to observe thin scattering layers. Extensive simulations of the performance of the GLAS lidar have been carried out based on actual cross section data obtained from our high altitude ER-2 aircraft lidar measurements (Spinhirne and Palm, 1997). These and other analysis indicate that GLAS will meet the stated measurement requirements. A factor that is not realistically included in the simulations is the much larger effect of multiple scattering for a spaceborne measurements (Spinhirne, 1982). As shown in calculations, the effective penetration into dense clouds is enhanced due to forward scattering. The GLAS instrument is designed to meet the stated measurement requirements under all conditions. The worst case conditions for lidar measurements are when there is a bright solar background, for example when the background is a dense lower cloud or snow and there is a high sun angle. The narrow band filtering and small field-of -view of the GLAS atmospheric channel is designed to provide adequate measurements in these worst conditions.

The GLAS instrument is a nadir viewing laser radar system. The transmitters are diode pumped $\mathrm{Nd}$ :YAG lasers operating at a fundamental and doubled wavelength. The wavelength, pulse rate and other parameters are listed in table 2

Table 2. GLAS instrument parameters

\begin{tabular}{|l|l|l|}
\hline PARAMETERS: & $.532 \mathrm{~nm}$ & $1064 \mathrm{~nm}$ \\
\hline Laser Pulse Energy & $36 \mathrm{~mJ}$ & $74 \mathrm{~mJ}$ \\
\hline Laser PRF & $40 \mathrm{~Hz}$ & $40 \mathrm{~Hz}$ \\
\hline Telescope Diameter & $0.9 \mathrm{~m}$ & $0.9 \mathrm{~m}$ \\
\hline Receiver FOV & $0.16 \mathrm{mrad}$ & $0.475 \mathrm{mrad}$ \\
\hline Optical Bandwidth & $25 \mathrm{pm}$ & $1.4 \mathrm{~nm}$ \\
\hline Detector Quantum Efficiency / Type & $0.6 /$ GAPD photon count & $0.3 /$ APD analog \\
\hline Range resolution & $75 \mathrm{~m}$ & $0.075 \mathrm{~m} \mathrm{\&} \mathrm{75} \mathrm{m}$ \\
\hline Pointing Knowledge & & 3 arcsec \\
\hline
\end{tabular}

There are three lasers, each rated for at least 2 years continuous operation, that would be used singly. The receiver is dual wavelength. The $1064 \mathrm{~nm}$ channel is the primary signal for surface ranging and from dense cloud tops. The $532 \mathrm{~nm}$ channel applies high efficiency GAPD solid state detectors for photon counting signal acquisition and is the primary channel for atmospheric signal acquisition. As stated an important aspect of the $532 \mathrm{~nm}$ atmospheric receiver channel is the small FOV and very narrow band filter to minimize the solar background noise signal and enable daytime observations. Since dense cloud signals will saturate the photon counting, the full atmospheric signal is also acquired with analog detection at $1064 \mathrm{~nm}$ and $75 \mathrm{~m}$ resolution. A high, $92^{\circ}$ orbit inclination for the GLAS mission permits good coverage of polar regions.

Spinhirne, J. D. and S. P. Palm, "Space based atmospheric measurements by GLAS,", in Advances in Atmospheric Remote Sensing with Lidar, A. Ansmann Ed., Springer, Berlin, 213217, 1996.

Spinhirne, J. D., M. Z. Hansen, and L. O. Caudill, "Cloud Top Remote Sensing by Airborne Lidar," Appl. Opt., 21, 1564, 1982.

Spinhirne, J. D., "Lidar Clear Atmosphere Multiple Scattering Dependence on Receiver Range," Appl. Opt., 21, 2466, 1982. 